\title{
Small area variation in crime effects of COVID-19 policies in England and Wales
}

\author{
Samuel Langton (University of Leeds) \\ Anthony Dixon (University of Leeds) \\ Graham Farrell (University of Leeds)
}

\begin{abstract}
It is well established that COVID-19 policies to restrict movement induced widespread falls in many crime types internationally. Much less is known about variation between areas in how these changes occurred. This study uses k-means clustering to examine local area variation in police notifiable offences across England and Wales. It finds that crime in most areas remained stable, a small proportion of areas accounting for disproportionate change. These were typically city centers with plentiful pre-pandemic crime opportunities, dominated by theft and shoplifting offences. We explore potential implications for policy, theory and further research.
\end{abstract}

Keywords: COVID-19, clustering, k-means, crime decline, crime opportunity theory, pandemic.

Corresponding author: Graham Farrell, School of Law, University of Leeds, LS2 9JT United Kingdom.

Email: g.farrell@leeds.ac.uk.

Competing interests: The authors declare that they have no competing interests.

Funding: The work was funded by the UKRI open call for research on COVID-19 under grant ES/00445X/1 from the Economic and Social Research Council.

Availability of data and materials: The data are publicly available and retrievable from the open online data portal for England, Wales and Northern Ireland. Code to replicate data downloads, handling and analyses is openly available (https://anonymous.4open.science/r/46199882-cc5a-449d-9946-300b3132ab2e/). 


\section{Introduction}

Crime rate changes in response to COVID-19 movement restrictions have been widely documented. This includes studies of Australia (Andresen and Hodgkinson 2021; Payne, Morgan and Piquero 2020, 2021), Canada (Hodgkinson \& Andresen, 2020), China (Borrion et al., 2020; Dai et al. 2020), England and Wales (Halford et al. 2020; Langton et al. 2020, Office for National Statistics 2020, Dixon and Farrell 2021), Mexico (Balmori de la Miyar et al. 2021; Estévez-Soto, 2020), Sweden (Gerell et al., 2020), and the United States (Abrams 2020; Ashby, 2020a, 2020b; Campedelli, Aziani, et al., 2020; Mohler et al., 2020; Piquero et al., 2020, Stickle \& Felson 2020). The findings are largely consistent with crime opportunity perspectives and the mobility theory of crime during the pandemic (Halford et al., 2020). That is, legally-enforced restrictions on daily activities, mobility and social interactions reduced crime opportunities. As restrictions were relaxed, these opportunities reemerged, and crime began to 'bounce back' closer to levels expected without the global pandemic (Langton et al., 2021).

While existing studies have provided insight into the impact of lockdown and social distancing on crime, research has almost exclusively been undertaken using macro-level units of analysis, such as cities or countries. Less is known about the local drivers of the lockdown crime drops or the degree to which macro-level trends are masking geographic inequalities in victimization. Pre-pandemic studies examining the long-term crime declines in many countries comprising the international crime drop (van Dijk et al. 2012) found significant inequalities (Adepeju et al., 2021; Bannister et al., 2018; Ignatans \& Pease, 2015, 2016; McVie et al., 2020) including at fine-grained spatial scales (Andresen and Malleson 2010; Andresen et al. 2017, Curman et al., 2015). These studies would suggest that local areas are unlikely to have experienced lockdown crime trends in unison. Rather, we might expect specific places, typically associated with high ambient populations and plentiful opportunities for crime (Malleson and Andreson 2015, 2016), to have driven the wider trend, with most local areas remaining fairly stable.

Examining the spatial distribution of the lockdown crime drop (and subsequent resurgence) represents the primary motivation of this paper. We decompose the macro-level trend in police-recorded crime observed in England and Wales between February and August 2020. We deploy non-parametric longitudinal clustering to identify clusters of meso-level units which contributed disproportionately to the nationwide drop and subsequent resurgence in crime during the nationwide lockdown. The spatial patterning, opportunity structure and crime type profile of these local areas are quantified and summarized for their consistency with expectations from opportunity theories of crime.

Relatively little is known about the spatial variation and local drivers of the macro-level trends. To what extent have local areas experienced lockdown trends in unison? This exploration represents a unique test of opportunity perspectives on crime, which would stipulate, for instance, that only a small number of local areas will have driven the lockdown crime drop. Prior to the COVID-19 pandemic, longitudinal studies of crime trends have consistently demonstrated that, in a changing macro-level scenario, most meso or micro-level areas remained remarkably stable. Instead, a disproportionately large volume of the macrolevel change is attributable to a small number of units (e.g.Adepeju et al., 2021; Andresen et al., 2017a, 2017b; Bannister et al., 2018; Curman et al., 2015; Trickett et al. 1995). This finding can be credited to highly localized opportunity structures, which can differ considerably within the same city, and even within the same neighborhood (Eck et al., 2000; Andresen and Malleson 2011). A shift in these local structures, brought about either through 
intervention (e.g. hotspot policing) or rapid changes in routine activities (Griffiths \& Chavez, 2004) can bring about wider (macro) change in crime rates, even if most units nested within the macro region remain stable.

In a lockdown scenario, we might expect these effects to be both exaggerated (in scale) and more instantaneous (in time). For instance, public transport hubs typically act as major crime generators due to the vast congregation of ambient populations in time and space facilitating the convergence of motivated offenders and suitable targets (Newton, 2018). In lockdown, public transport services in some countries including England and Wales were either closed completely, or open only for a limited number of essential purposes. With the necessary convergence of offenders and victims disrupted, often quite literally overnight, we would expect these customarily high crime places to fall considerably, prompting a macro-level decline. By contrast, areas typically devoid of crime opportunity, with say, little or no ambient populations, may remain largely unaffected by lockdown restrictions on mobility, and in turn, contribute little to any macro-level change. In other words, while lockdowns have often been imposed equitably at a city or national level, the effect on crime will likely be moderated by the opportunity structure of local areas.

Preliminary evidence from the United States certainly suggests that this may be the case. Using police-recorded crime data in San Francisco and Oakland, Shayegh \& Malpede (2020) provided visual descriptive evidence which indicated a degree of geographic variation in pre and post-lockdown crime. In Detroit, a study using a small sample of block units found that, amidst a fall in burglaries following stay at home orders, there was a shift in concentrations away from residential areas towards mixed and non-residential parts of the city (Felson et al., 2020). In Chicago, there was evidence of variability in the extent to which lockdown policies impacted upon crime. A small proportion of communities drove the citywide decline, with most areas remaining largely unchanged, and some areas even increasing, bucking the macro-level trend entirely (Campedelli et al., 2020). Using regional units of analysis nested within the state of Queensland, Australia, Payne et al. (2021) found a degree of diversity in crime rate trends, suggesting that the lockdown crime drop was not 'universal'.

In England and Wales, early descriptive evidence from the first three months of lockdown suggests that previously high-crime areas may have experienced the steepest relative declines compared to previous years (Dixon et al., 2020). Increases in fly-tipping have also been linked to a small number of councils, with trends varying considerably between regions (Dixon \& Tlley, 2020). However, there has not been a comprehensive decomposition of local longitudinal variation underpinning lockdown crime drops, or indeed an exploration of the opportunity structures characterizing the local areas which have driven macro-level changes.

In this paper, we aim to identify and describe the localized drivers of the lockdown crime drop in England and Wales. We achieve this using 7-months of police-recorded crime data between February and August, 2020. First, we summarize the national (macro) trend in terms of crime counts in comparison to previous years. Second, using a non-parametric longitudinal clustering technique, we identify meaningful clusters of meso-level areas which unpick stable (and volatile) local areas underpinning the macro-level trend. Third, we describe the clusters identified in terms of their spatial patterning, opportunity structures, and crime type profile.

\section{Data and Method}

To examine localized instability in the lockdown crime drop, we make use of three data sources, namely, open police-recorded crime data, geographic boundaries from Ordnance 
Survey and the Office for National Statistics (ONS), and data sourced from the Open Street Map API. Each of these are now outlined in turn, followed by an outline of the methods deployed. Code to replicate the data downloads, handling, analyses and visualization reported here are openly available (https://anonymous.4open.science/r/46199882-cc5a-449d-9946$300 \mathrm{~b} 3132 \mathrm{ab} 2 \mathrm{e} /)$.

\subsection{Crime data}

Open police-recorded data on crime and anti-social behavior in England and Wales is published through an online web portal (https://data.police.uk/). Individual records are released on a month-by-month basis for each of the 43 police forces comprising England and Wales. We used a study period spanning February to August 2020 in order to capture the first six months of the nationwide lockdown (March to August) and the one month preceding the change (February). Here, we note that lockdown was initiated on 23 March, making April the first full month of measures. For reference and comparison to historical trends, we obtained data for the same months in 2018 and 2019. Individual records are time-stamped by month the temporal scale of this study. Due to incomplete data releases from Greater Manchester Police, we excluded data from the Greater Manchester region, collating data from 42 out of 43 forces in England and Wales.

Individual open records categorize crime according to thirteen different notifiable offence categories (e.g. burglary, violence and sexual offences, theft from the person, vehicle crime). ${ }^{1}$ Records also include anti-social behaviour (ASB) which usually captures less serious offences such as nuisance behavior and is not a notifiable offence. Individual records were aggregated to create a count measure for 'notifiable offences (excluding drugs)' by month at the nationwide (macro) level, and the localized (meso) level, as detailed in the next section. The decision to exclude drug offences follows widespread recognition that drug crime trends, particularly during the COVID-19 lockdown, largely reflect policing proactivity rather than meaningful shifts in criminal behavior (Langton \& Solymosi, 2020). Recognizing that aggregating data across crime types can mask variation (Andresen et al., 2017) we later decompose our main findings according to the twelve remaining notifiable offences, and report additional analyses broken down by crime type in the Appendix.

\subsection{Unit of analysis}

To provide national context to the main analysis, we use the (macro) geographic region of England and Wales, noting the exclusion of Greater Manchester. For localized (meso) analysis, we aggregate offences to Lower Super Output Area (LSOA). LSOA are a mesolevel geographic units designed for the reporting of official statistics at small geographies (Office of National Statistics, 2021). England and Wales is comprised of 32,844 LSOA designed to be uniform by resident population size. In 2019, the average LSOA housed 1,700 people. Data obtained from the open police portal (see previous section) include a preassigned field stating the LSOA in which the crime occurred as recorded by the police. Due to the spatial anonymization method used prior to data release, LSOA are the lowest level of aggregation at which we can reasonably assume spatial accuracy across multiple crime types (Tompson et al., 2015). LSOA boundaries reflect 'on the ground' characteristics such as roads and railways (Office of National Statistics, 2012), and as such, we consider LSOA to represent meaningful behavioral spaces to study the opportunity structures of crime. After

\footnotetext{
${ }^{1}$ Further information on these crime categories can be obtained from https://www.police.uk/pu/contact-thepolice/what-and-how-to-report/what-report/.
} 
removing crimes recorded by Greater Manchester Police, crimes recorded to have occurred within the Greater Manchester region, and four LSOA which contained no crime between 2018 and 2020 (likely due to the spatial anonymization process), our final sample for the meso-level analysis comprised 33,075 LSOA.

\subsection{Open Street Map}

To summarize the opportunity structure of local areas we required a nationwide dataset of theoretically relevant facilities and urban features which could be aggregated at the LSOA level. To this end, we obtained point-level data from the Application Programming Interface (API) for Open Street Map via the osmdata package (Padgham et al., 2017) in $R$ (R Core Team, 2013). Open Street Map is a crowdsourced geospatial database containing a vast array of features which can be used for explaining the temporal and spatial patterning of crime (Langton \& Solymosi, 2020). Geographic features are identified by pairs of keys and values which can be used to computationally query the API for geospatial data. Based on existing research examining the opportunity structures of fine-grained spatial scales, we collated the coordinate locations of the following facilities:

- Nightlife: pubs, nightclubs, restaurants.

- Shops: convenience stores, malls, shoe shops, department stores, clothes shops, electrical shops, supermarkets, chemists, greengrocers.

- Public transport: bus stops and railway stations.

- Bicycle parking: bicycle parking lots.

The point-level data on these features were aggregated to create counts for each facility by LSOA. For simplicity, and due to issues of data sparsity, we sum the counts for each facility according to their overarching description (i.e. nightlife, shops, public transport, bicycle parking). We expect that LSOA containing a high number of facility counts across each domain will have higher pre-pandemic levels of crime, due to the plentiful opportunities for crime, and in turn, steeper declines in crime following lockdown as a result of these opportunities suddenly becoming unavailable. We expect areas with low counts across these domains to have similarly low crime levels pre-lockdown, and thus will remain low and stable following lockdown commencement.

\section{Method}

Analyses to examine the localized variation in the lockdown crime drop are conducted in three principal stages. First, an overview of the nationwide (macro) trend is provided in terms of absolute counts. Second, the macro-level trend is disentangled using non-parametric clustering techniques on the LSOA (meso) units $(\mathrm{N}=33,075)$. Third, the characteristics of each cluster are summarized in terms of their opportunity structures, spatial patterning and crime type profile. Each of these steps is now outlined in turn.

\subsection{Macro-level descriptives}

Macro-level descriptives of count trends notifiable offences (excluding drugs) are reported to provide the context from which we will unmask local (meso) variation. We visualize observed counts between February and August 2020 relative to the same periods in 2018 and 2019. In doing so, we can observe how crime trends changed in the face of lockdown measures in England and Wales (see also Langton et al. 2021). This sets the scene from which we can disentangle the underlying meso-level variation. 


\subsection{Meso-level clustering}

To quantify the degree of meso-level uniformity underpinning the macro-level trend, and identify the potential drivers of the lockdown crime drop (and resurgence), we deploy a longitudinal variant of k-means clustering (Genolini, Alacoque, Sentenac, et al., 2015b; Genolini \& Falissard, 2011). This non-parametric clustering technique has an established role in crime and place research for examining the longitudinal trajectories of local areas in a macro-level crime drop scenario (Andresen et al., 2017; Curman et al., 2015).

The natural experiment conditions of the COVID-19 lockdown (Felson \& Stickle 2020) make the usage of k-means particularly suitable. Existing research adopting the method has tended to investigate long-term change over years or decades, focusing on the directional homogeneity (e.g. increasing, decreasing or stable) of clusters, rather than short-term volatility (Andresen et al., 2017). To this end, it has demonstrated comparable value to model-based techniques such as group-based trajectory modelling (Curman et al., 2015). But, a key strength of k-means is that it is also capable of identifying short-term fluctuation in longitudinal trends (Adepeju et al., 2021). In the lockdown scenario, crime opportunities were withdrawn quite literally overnight, and thus we might expect a similarly rapid and short-term change in crime at meso spatial scales. The ability to unpick these rapid changes represents a key strength of k-means over non-parametric techniques such as anchored k-medoids, which are designed for long-term rather than short-term change (Adepeju et al., 2021) or groupbased trajectory modelling, which is limited by polynomial terms (Griffiths \& Chavez, 2004).

We deploy k-means using the $\mathrm{kml}$ package (Genolini, Alacoque, Sentenac, et al., 2015a) in R on notifiable offence (excluding drug) counts for LSOA in England and Wales ( $\mathrm{N}=33,075)$ between February and August 2020. To achieve a parsimonious cluster solution while minimizing the risk of missing underlying variation, we proposed potential solutions between two and eight clusters, choosing the final solution based on the Calinski-Criterion (Caliński \& Harabasz, 1974). For each potential solution, twenty redraws with different starting conditions were run to ensure that solutions were stable. Potential cluster solution options were also examined using Principal Component Analysis to establish their suitability.

We visualize the final cluster solution in a manner which conveys the underlying distribution of observations comprising each cluster at each time point, rather than reporting a summary statistic (e.g. the mean trajectory) in isolation. For each cluster, we also overlay the equivalent trajectories for 2018 and 2019 as a reference point for comparison to a 'typical' year. In doing so, we aim to not only identify localized (in)stability in the lockdown crime drop, but also assess the extent to which the trends observed have deviated from previous years.

\subsection{Cluster characteristics}

We hypothesize that the meso-level areas driving the lockdown crime drop will be those with plentiful opportunities for crime. That is, a disproportionately large volume of the decline (and subsequent resurgence) will be attributable to a handful of places which had pre-existing high crime levels as a result of their opportunity structure. Using the measures for opportunity generated from Open Street Map (i.e. nightlife, shops, public transport and bicycle parking), we report descriptive statistics on facility counts for each of the clusters obtained from the k-means analysis. In doing so, we expect to unpick a meaningful pattern which is consistent with the opportunity perspective of crime. 
To supplement this, we visualize the spatial patterning of the cluster solutions. For brevity and simplicity, we focus on Birmingham, Liverpool, Leeds, Bradford, Sheffield and Cardiff. We have excluded Greater Manchester due to the lack of police data, and given its size, we determined Greater London to warrant an individual case study for future research. Given these exclusions, the six cities we report represent the five most populous cities in England, and the most populous city in Wales. Study regions are defined by based on the city names appearing in LSOA name. For the purposes of the visual, one LSOA in Cardiff containing Flat Holm Island, which is off the coast, was been removed.

Finally, recognizing the unique opportunity structure of specific crimes, we summarize the crime type profile of clusters. For each cluster, we report the percentage breakdown of crimes types. We suspect that clusters will have differing crime type profiles according to the opportunity structures of each grouping.

\section{Results}

\subsection{Nationwide trends}

To set the context for the localized analysis, Figure 1 visualizes crime counts between February and August 2020 for notifiable offences excluding drugs before and after lockdown. In April, the first full month of lockdown in England and Wales, we observe a nationwide decline in notifiable offences in comparison to previous years. Upon the relaxation of lockdown rules, crime began to bounce back, and by August, crime had returned to within a range we might have expected without the nationwide lockdown (see also Langton et al., 2021). This trend represents the 'global' trend which we will subsequently disentangle using localized analyses.

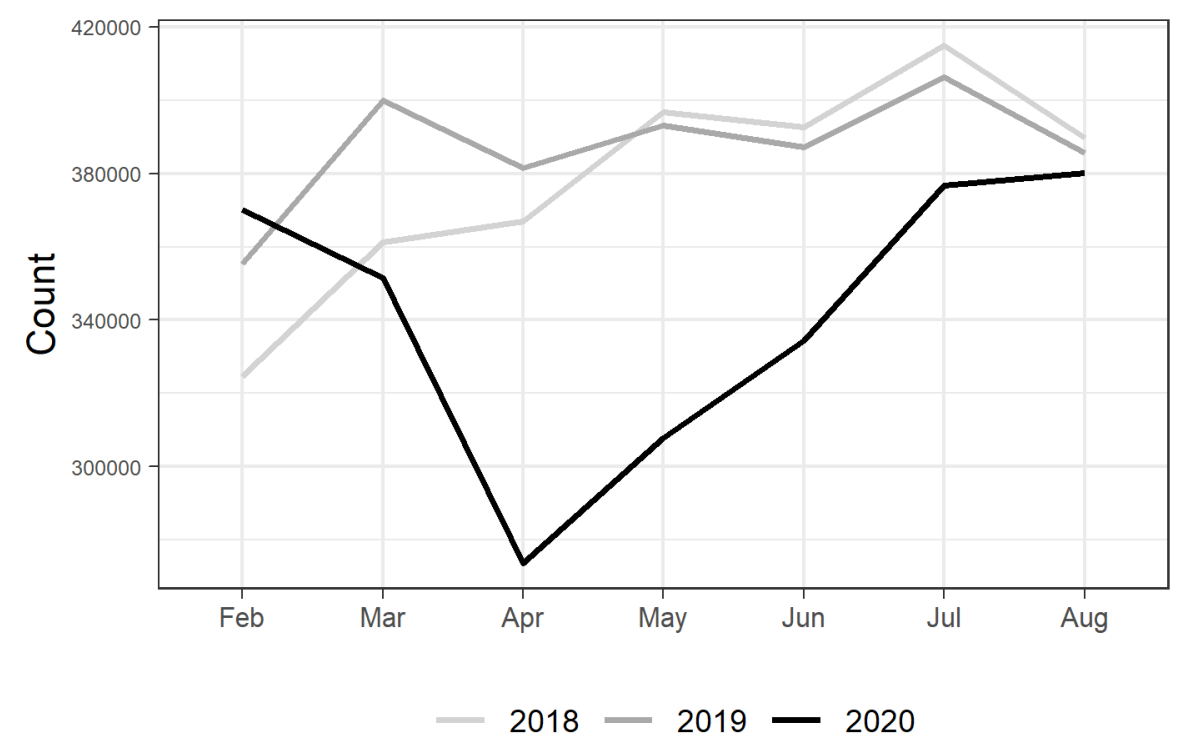

Figure 1: Notifiable offence (excluding drugs) end of month counts in England and Wales. April was the first full month of lockdown. 


\subsection{Longitudinal clustering}

\subsubsection{Cluster trends}

We deploy non-parametric k-means clustering on LSOA (meso) level geographic units $(\mathrm{N}=$ 33,075) comprising England and Wales to decompose the macro-level 'decline and resurgence' observed between February and August, 2020. Based on an assessment of the Calinski-Criterion statistic (Caliński \& Harabasz, 1974) and Principal Component Analysis, we determined the optimal solution to be 6 clusters (see Figure 2). Solid black lines represent the median count for each cluster at any given time point, with the black dotted line showing the mean. Violin plots have been added to convey variation around these points at each time point. These suggest that the mean and median point statistics summarize the underlying data reasonably well, and indicate that clusters are distinct from one another, with little overlap. Additional lines have been added to convey each clusters' mean and median trend in 2018 and 2019 respectively. These trends suggest that the clusters identified using the 2020 study period were distinct and meaningful even in previous years, and provide a relative baseline from which we can compare lockdown trends.

Overall, we note that most LSOA were remarkably stable during the pandemic. Clusters A and B could be described and 'low crime and stable', exhibiting fairly low counts throughout the study period and across years. Together, these clusters comprise $89 \%$ of LSOA in England and Wales. Even amidst the stark macro-level decline in notifiable offences (see Figure 1) LSOA in these clusters only experienced marginal average dips in crime. The third largest cluster, cluster C, comprises 10\% of LSOA in England and Wales. LSOA in this cluster experienced a more prominent dip in crime, along with cluster D (1.6\% of LSOA). Together, we might describe these LSOAs as 'mid-crime, mid-drop'. In both cases, there is a clear deviation from previous years. Notifiable offences fall between March and April, and then began to converge back to levels observed in previous years.

That said, most 'action' appears to be occurring amongst a small subset of LSOA. Clusters E and $\mathrm{F}$ collectively comprise only $0.33 \%(\mathrm{~N}=110)$. Yet, their crime counts are much higher, and the decline between March and April is considerable. We might therefore describe these clusters as 'high crime, major drop': LSOA with plentiful opportunities for crime in typical times, and in turn, LSOA which are most sensitive to the restriction in opportunities which followed after the imposition of lockdown. To further decompose these clusters, we now disentangle the contribution of each cluster. 

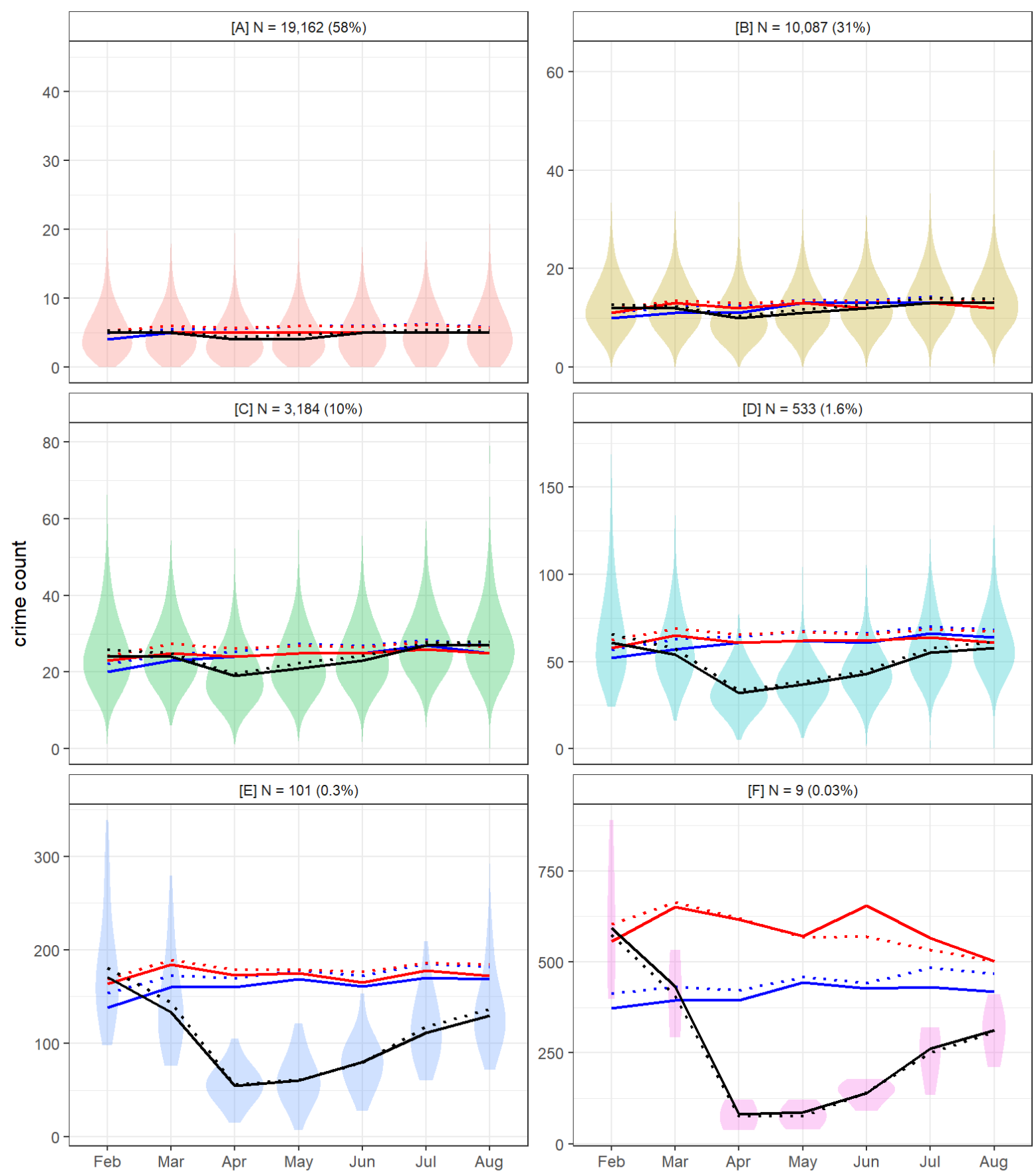

- $2020-2018-2019$

Figure 2: K-means cluster solutions for LSOA notifiable offences (excluding drugs).

Distributions refer to 2020 only.

\subsubsection{Contribution of each cluster}

To further unpick the contribution of these clusters to the nationwide lockdown crime drop, Figure 3 plots the monthly change in counts and percentage of total absolute change (i.e. positive or negative) attributable to each cluster. We can use this visual to identify which clusters drove the initial decline and subsequent nationwide resurgence in crime. 
As expected, the vast majority of change across all clusters occurred between March and April. Between these months, notifiable offences experienced a dramatic fall nationwide. That said, the figure demonstrates that this decline did not occur equitably across local areas. Consistent with the cluster solutions trends in Figure 2, a small number of LSOA (meso) units appear to have contributed disproportionately to the nationwide (macro) trend. For instance, clusters A and B, which comprise $58 \%$ and $31 \%$ of LSOA in the country, accounted for only $20 \%$ and $27 \%$ of the total decline between March and April. By contrast, clusters E and $\mathrm{F}$, which together comprised only $0.33 \%$ of LSOA $(\mathrm{N}=110)$ contributed to $15 \%$ of the nationwide drop in these months. That is, crime in most areas was remarkably stable, given the nationwide volatility, with just a handful of areas driving the decline the nationwide lockdown crime drop.

This picture shifts slightly upon the beginning of the resurgence between April and May. Clusters A and B continue to contribute a disproportionately small amount to nationwide change, but the proportion increases, with each contributing $24 \%$ and $42 \%$ to the increase respectively. The contribution of the smallest clusters $\mathrm{E}$ and $\mathrm{F}$ is small compared to the initial decline, $2 \%$ and $0 \%$ (when rounded), respectively. This can be largely attributed to the flattening of their crime count trends between these months. In other words, following their initially steep decline between March and April, crime remained reasonably unchanged between April and May in the 'high crime, major drop' areas.

Following the period of stabilization, clusters $\mathrm{E}$ and $\mathrm{F}$ gather pace during the resurgence, as does cluster D. For instance, between June and July, these three clusters collectively account for $27 \%$ of the nationwide increase, despite comprising less than $2 \%$ of LSOA in the country. July to August is somewhat different. Until that point, each cluster, despite their vastly differing relative contributions to change, were directionally homogeneous. In other words, every cluster declined between February and April, and then increased between April and July. Between July and August, clusters began to diverge. Nationwide, there was a marginal increase in notifiable offence counts (see Figure 1), but this clearly masks a great deal of localized variability.

The 'low crime and stable' clusters (A and B) actually declined again between July and August, and the higher crime clusters continued to increase. The change is almost imperceptible when viewing average counts in these LSOA (see Figure 2) but the sheer size of these units, collectively comprising $89 \%$ of England and Wales, had a major impact on nationwide trends. This is suggestive of highly localized change in opportunities as lockdown rules were relaxed: change which is aggregated away at the macro-level. Stable, low crime areas mitigated against further nationwide increases which may, if they had continued, resulted in a higher crime count in August than observed in previous years. 


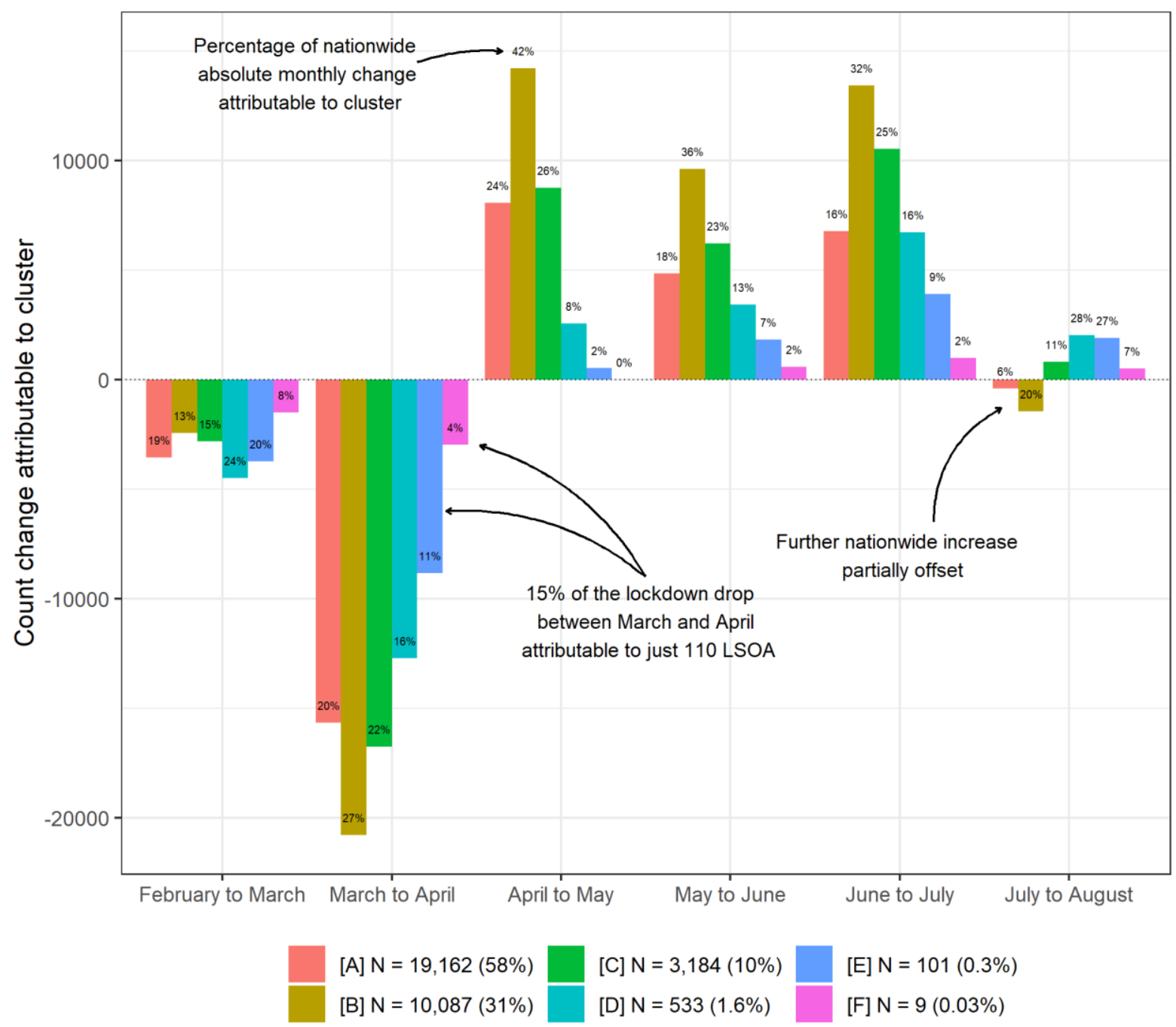

Figure 3: Counts and percentage of nationwide change between months attributable to each cluster.

These findings can be supplemented with a comparison to the previous year. Figure 4 compares the clusters identified in 2020 with the equivalent months in 2019 . Here, it is clear that the 'high crime major drop' clusters (E and F) contributed the most to the lockdown crime drop relative to their size. By way of example, around 27,000 fewer crimes occurred in cluster A-containing 19,162 LSOA-during April 2020 compared to April 2019 (Figure 4a). This translates to a $24 \%$ drop (Figure $4 \mathrm{~b}$ ), and an average fall per LSOA of 1.39 counts (Figure 4c). For cluster F, containing just 9 LSOA, approximately 5,000 fewer crimes occurred (Figure 4a), which translates to an 87\% drop (Figure 4b), and an average fall per LSOA of 543 counts (Figure 4c). In other words: whether comparing month-to-month change in 2020, or comparing equivalent months in 2019, the lockdown crime drop was disproportionately driven by a small number of local areas, with most meso-level units remaining fairly stable, even amidst dramatic macro-level change. 

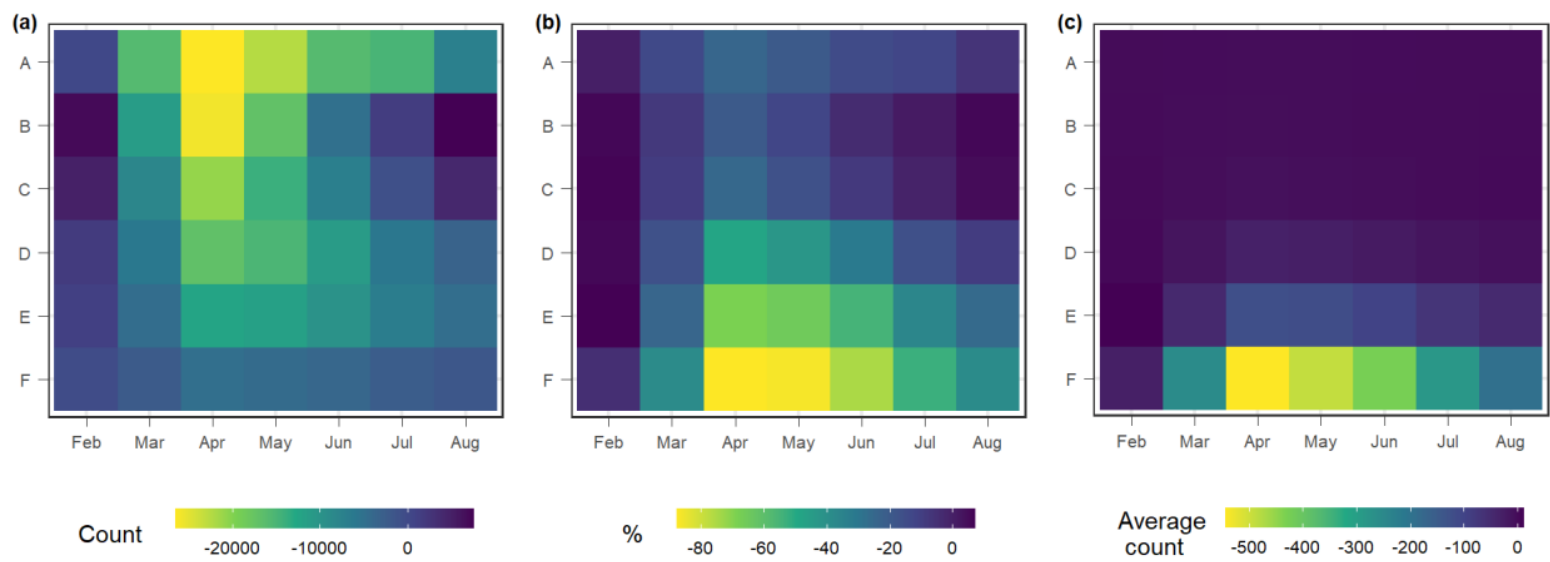

Figure 4: Comparing LSOA clusters between equivalent months in 2020 and 2019 according to (a) absolute difference in counts, (b) percentage difference in counts, (c) average difference in counts.

\subsection{Characteristics of clusters}

\subsubsection{Spatial distribution}

To provide local context to the cluster solutions identified through the k-means clustering, we visualize the spatial patterning of groupings for six major cities in England and Wales (see Figure 5). We find distinct geographic patterns to the clusters identified. The maps denote a single 'city center' based on the LSOA with the highest count of Open Street Map features (i.e. the sum of nightlife, shops, public transport and bicycle parking). Because LSOA are uniform by residential population, geographically small LSOA tend to be areas with higher population density. Here, it is worth noting that the Bradford region contains the city of Bradford, but also the satellite town of Keighley to the north west.

Without exception, the city centers and commercial districts of each city are characterised by 'high crime, major drop' clusters $\mathrm{E}$ and $\mathrm{F}$, which in turn often neighbor the 'mid crime, mid drop' cluster D. By contrast, the 'low crime and stable' clusters tend to sit outside of the city centres: cluster B in the suburbs and the sparsely populated cluster A on the periphery of each urban conurbation. As such, changes in crime during lockdown appear to have a distinct geographic pattern: the nationwide drop was not experienced uniformly across space, but rather, particular areas including city centers appear to have driven the macro-level trend, with outer suburbs remaining fairly stable. For a detailed and interactive investigation of the spatial clusters identified for the whole of England and Wales, we refer readers to an openly available online map (anonymous for review). 

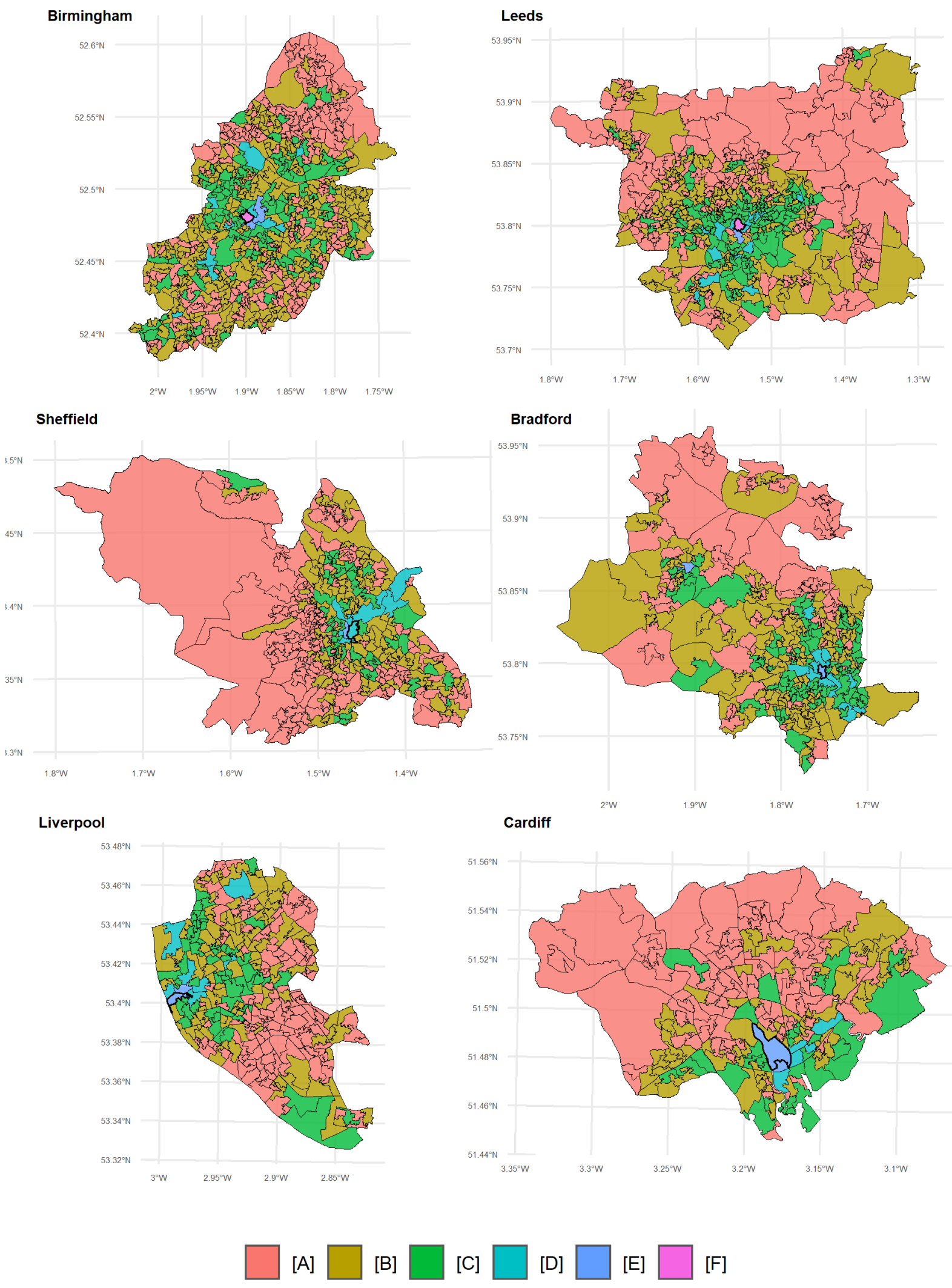

Figure 5: Spatial distribution of clusters by major urban conurbation. LSOA with the highest count of Open Street Map features (in black) denotes city centre. 


\subsubsection{Opportunity structure}

We have now identified that, amidst dramatic nationwide change in notifiable offences during lockdown, there has been considerable underlying volatility, with a small number of local areas driving the macro-level trend. From a theoretical perspective, we expect the clusters to have differing opportunity structures. Table 1 reports a series of descriptive statistics based on the opportunity structure of each cluster using the nationwide data obtained from the Open Street Map API. These findings largely support our expectations, namely, that large, stable clusters have few opportunities for crime, while high crime areas which were responsible for a disproportionately large amount of the crime drop contain plentiful features which facilitate crime.

By way of example, there are sparse opportunities for crime in the largest and most stable cluster A. Most LSOA in this cluster have no nightlife facilities, no shops and no bicycle parking. Public transport is available but it is not common: the median LSOA in cluster A only contained four bus stops or railways stations. As we move along to medium and high crime clusters, which had higher pre-existing crime levels, and thus steeper declines during lockdown, these counts markedly increase. For instance, LSOA in cluster E contain a median of 21 nightlife facilities, 28 shops, 24 public transport nodes and 13 bicycle parking spaces. These counts increase further for cluster F, although we note that the cluster contains only nine LSOA. This is consistent with the findings presented earlier which suggest crime declined the most in these clusters: areas previously rich in opportunity, in which crime was pervasive, became the drivers of the lockdown crime drop.

[Insert Table 1 here. Caption: Descriptive statistics of facilities and features in each cluster. Sourced from Open Street Map.]

\subsubsection{Crime type profile}

Given the unique longitudinal trends, spatial patterning and opportunity structure of the clusters identified using the aggregate notifiable offences measure, we might expect the clusters identified to have distinct crime type profiles (see Figure 6). In February, before the restrictions on mobility and social interaction, the crime type profiles of each cluster were already distinct. Higher crime clusters (e.g. E, and F) were weighted heavily towards shoplifting and theft. Low crime and stable clusters, by contrast, had higher proportions of criminal damage and arson, burglary and violence and sexual offences.

Overall, we observe a degree stability in the distribution of crime types both within and between clusters. The largest, stable clusters (A and B) have remarkably similar crime type profiles. Before lockdown, for each of these clusters, the most prevalent crimes were violence and sexual offences, both comprising $41 \%$ of total crime. This proportion increased on lockdown commencement, largely at the expense of burglary and vehicle crime, which decreased as a proportion of total crime. In clusters A and B, and indeed across all clusters, the proportion of total crime attributable to public order also increased. These increases may reflect a seasonal effect: crimes such as violence and sexual offences and public order tend to increase between February and August in typical times, while vehicle crime and burglary are usually stable (Langton et al. (2021); see Appendix). This does not, by any means, indicate that these crimes increased during lockdown - counts declined considerably - but rather, these crimes declined less steeply relative to other offence categories. In the case of public order, this may be a result of lockdown-specific activity. Public order includes offences relating to processions and assemblies, and thus may capture gatherings which violated COVID-19 guidelines (Crown Prosecution Service, 2021) and protests such as those relating 
to Black Lives Matter which were prominent in summer 2020 (Baggs 2020). Increases in public order offences across these areas would slow and work against a fall in a more typical public order crimes.

Notable disparities between clusters arise with regards to theft from the person and other theft in cluster $\mathrm{F}$, which as reported earlier, drove a considerable part of the nationwide decline in crime. Theft in these LSOA was squeezed upon lockdown commencement. In February, theft crime types comprised 53\% of total crime, but by April, this figure had dropped to $13 \%$. This occurred despite these crime types typically remaining stable during equivalent times of year (Langton et al. (2021); see Appendix). Somewhat surprisingly, given the spatial distribution and opportunity structures of clusters $\mathrm{E}$ and $\mathrm{F}$ in city centres, shoplifting did not experience a similar squeeze in these LSOA.
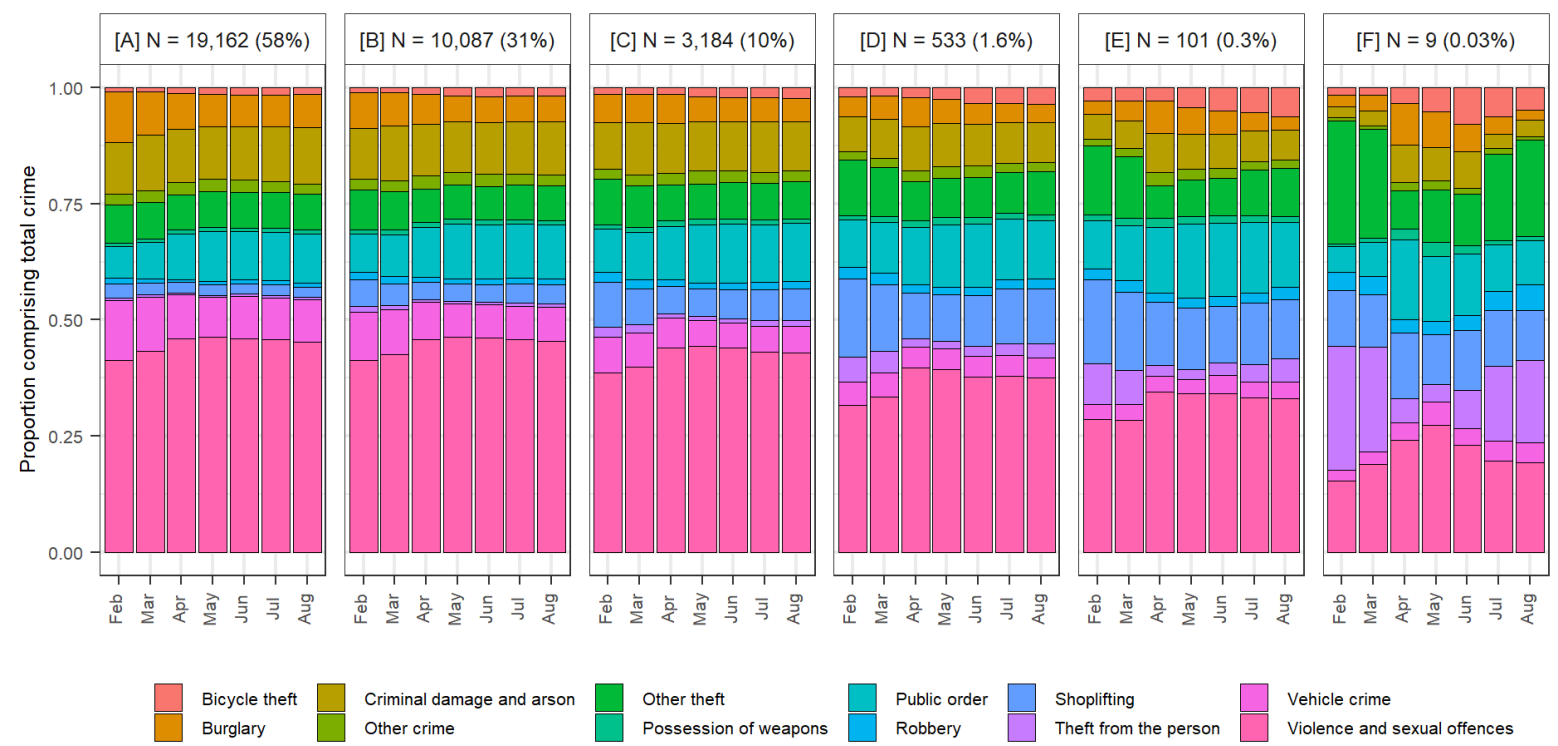

Figure 6: Crime type profiles of each cluster solution.

\section{Discussion}

The finding that a small number of areas drove the national crime trends, with most areas otherwise remaining fairly stable, is consistent with other studies. Evidence from Queensland, Australia (Payne et al., 2021) and Chicago, United States (Campedelli, Favarin, et al., 2020) suggests that the aggregate lockdown crime drop masked underlying variation. Likewise, studies from before the pandemic found aggregate crime trends largely attributable to variation in a handful of areas (Andresen et al., 2017; Curman et al. 2015; Ignatans and Pease 2015, 2016;). Here, for the first time, we have exploited the quasi-'natural experiment' conditions of the pandemic to decompose this variation during both the decline and resurgence stages of a macro-level trend, over a short period of time.

Findings suggest that the macro-level effect of lockdown on crime has been moderated by highly localized characteristics. Previously criminogenic areas, characterised by plentiful opportunities for crime, and often located in city centers, were the most susceptible to decline following the introduction of "stay at home" measures. Containing a disproportionately large number of nighttime economy facilities (e.g. pubs, nightclubs), commercial outlets (e.g. malls, convenience stores), public transport nodes (e.g. railway 
stations, bus stops) and bicycle parking spaces, these areas were where crime was typically concentrated. In March, overnight, as the hospitality sector closed its doors, non-essential shops closed, and transport either closed or became unnecessary, these key features became unavailable or unusable. In turn, these areas contributed a considerable (and disproportionate) amount to the nationwide decline in crime.

That being said, it is notable that the local drivers of the lockdown crime drop experienced only a modest resurgence upon the easing of lockdown restrictions. By August, the clusters identified as 'high crime major drop' remained well below the crime counts observed in 2018 and 2019, by which point the low and mid-crime clusters had returned to historical levels. This can likely be attributed to crime type profile of clusters. The drivers of the lockdown crime drop were weighted heavily towards theft (theft from the person, other theft) and shoplifting compared to the low and mid-crime clusters. Instead, low and mid-crime areas were characterized by a larger proportion of violence and sexual offences. Breaking nationwide trends down by crime type, we observe starkly different resurgence trends across these crime types. Whereas violence and sexual offences had returned to expected levels by August, theft and shoplifting had not. This is evidenced by a descriptive comparison to 2018 and 2019 (see Appendix) and more advanced forecasting techniques which account for longterm trends (see Langton et al., 2021). This may reflect the asymmetric manner of lockdown in England and Wales. While it came into force instantaneously and equitably across the country, lockdown was lifted in stages. For instance, a select number of commercial outlets (e.g. gardening centers) were reopened in May, but restrictions on the hospitality sector and public transport remained in place until July.

Here we would note some weaknesses in the study. First, the crime measure of 'notifiable offences (excluding drugs)' aggregates away some detail in longitudinal trends. The crime type profile findings certainly go some way in addressing this, and we have now set a baseline from which further investigations can be conducted. However, further insight could be gained from replicating this analyses for major crime types which we know to have distinct opportunity structures, such as burglary and theft. Here, we would note that the generalized Gini coefficients by crime type (see Appendix) certainly suggest that there may be differences between crime types, as have preliminary findings using decile clusters (Dixon et al., 2020). Secondly, the Open Street Map API used to quantify the prevalence of crime opportunities in each cluster is imperfect. Studies have found an association between contributions to Open Street Map and contextual characteristics such as poverty and distance from the city center - factors that are also known to be associated with crime. Due to computational issues and API query limits, some features were also unobtainable on a national scale, such as the residential buildings footprints. While we would argue that Open Street Map is unique in its ability to offer open data on opportunity structures nationwide, we would encourage future research to verify these findings using other data sources.

\section{Conclusion}

This paper investigated spatial variation in the COVID-19 crime drop in England and Wales. Using 7-months of police-recorded data on notifiable offences (excluding drugs), we decomposed the nationwide decline in crime according to clusters of local areas, each with distinct longitudinal trajectories. Findings provide substantial evidence to suggest that the nationwide decline in crime during lockdown was driven by a disproportionately small number of areas. We find that these areas, predominately city centers, had pre-existing high levels of crime, likely attributable to their unique opportunity structures, containing numerous 
nightlife facilities, commercial shops, public transport nodes and bicycle parking spaces. Clusters demonstrate a degree of stability in their crime-type profiles, but the 'high crime, major drop' areas appear to be weighted towards the volume crimes of theft and shoplifting. Despite their dramatic declines, these areas experienced a fairly shallow resurgence upon the relaxation of lockdown rules, remaining below historical levels even by the time low and mid-crime clusters had returned to expected levels. The findings lend weight to opportunity theories of crime and to a mobility theory of crime in the pandemic (Halford et al. 2020). Dramatic changes in crime during the pandemic occurred due to movement restrictions which impacted disproportionately upon crime in areas where it was previously concentrated. 


\section{Appendix}
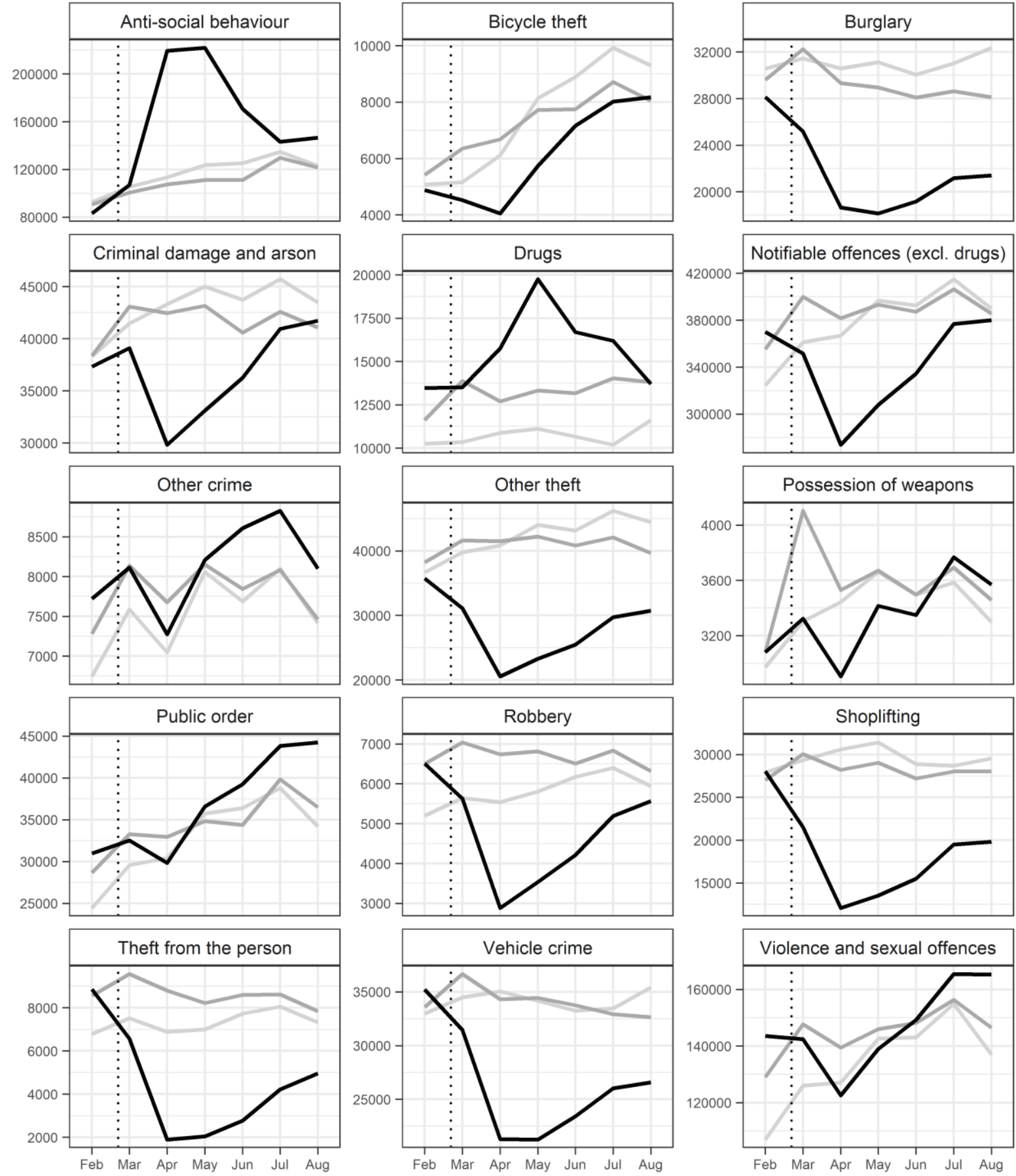

$-2018-2019-2020$

Figure 7: Counts by offence type in England and Wales during the lockdown period. 

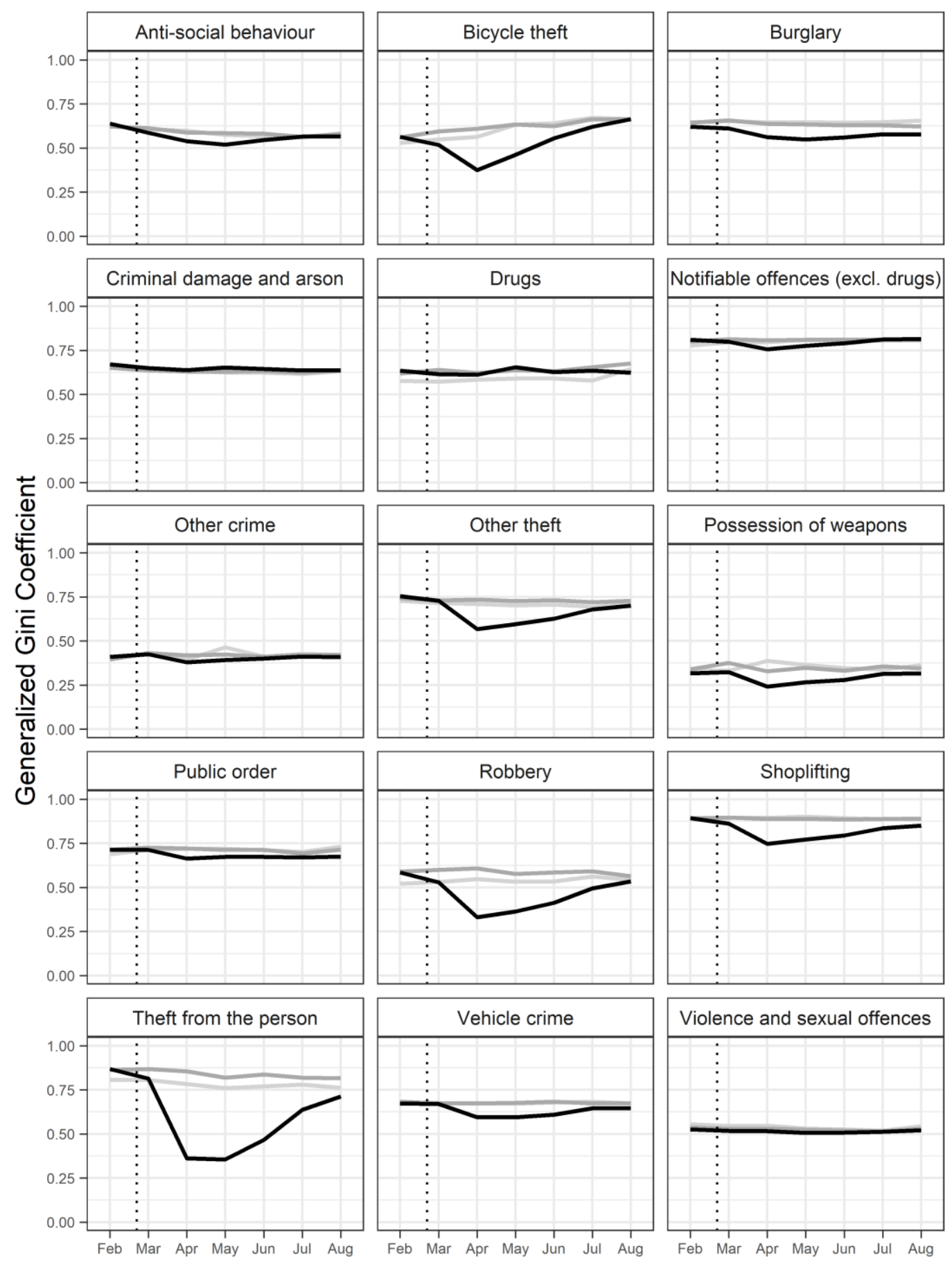

$2018-2019-2020$

Figure 8: Monthly generalized Gini coefficient trends by offence type in England and Wales during the lockdown period. 


\section{References}

Abrams, D. 2020. COVID and Crime: An Early Empirical Look, Research Paper No. 20-49, University of Pennsylvania Carey Law School, 14 August. University of Pennsylvania: Institute for Law and Economics.

Adepeju, M., Langton, S., \& Bannister, J. (2021). Nchored k-medoids: A novel adaptation of kmedoids further refined to measure long-term instability in the exposure to crime. Journal of Computational Social Science. https://doi.org/https://doi.org/10.1007/s42001-021-00103-1.

Andresen, M.A. and N. Malleson. 2011. Testing the stability of crime patterns: Implications for theory and practice, Journal of Research in Crime and Delinquency, 48(1); 58-82.

Andresen, M.A., Linning, S.J. and N. Malleson. 2017a. Crime at places and spatial concentrations: Exploring the spatial stability of property crime in Vancouver BC, 2003-2013. Journal of Quantitative Criminology, 33(2); 255-275.

Andresen, M. A., Curman, A. S., \& Linning, S. J. 2017b. The trajectories of crime at places: Understanding the patterns of disaggregated crime types. Journal of Quantitative Criminology, 33(3), 427-449.

Andresen, M.A. and T. Hodgkinson. 2020. Somehow I always end up alone: COVID-19, social isolation and crime in Queensland, Australia, Crime Science, 9(25); 1-20.

Ashby, M. 2020a. Initial evidence on the relationship between the coronavirus pandemic and crime in the united states. Crime Science, 9, 1-16.

Ashby, M. 2020b. Changes for police calls for service during the early months of the 2020 Coronavirus pandemic, Policing, 14(4); 1054-1072.

Balmori de la Mayir, J. R. L. Hoehn-Velasco \& A. Silverio-Murillo. 2021. Druglords don't stay at home: COVID-19 pandemic and crime patterns in Mexio city, Journal of Criminal Justice, 72, https://doi.org/10.1016/j.jcrimjus.2020.101745

Bannister, J., Bates, E., \& Kearns, A. (2018). Local variance in the crime drop: A longitudinal study of neighbourhoods in greater glasgow, scotland. The British Journal of Criminology, 58(1), 177-199.

Baggs, M. 2020. Black Lives Matter in the UK: 'We're still not being heard' BBC News online, 25 August 2020, at https://www.bbc.co.uk/news/newsbeat-53812576

Borrion, H., Kurland, J., Tilley, N., \& Chen, P. (2020). Measuring the resilience of criminogenic ecosystems to global disruption: A case-study of covid-19 in china. Plos One, 15(10), e0240077.

Caliński, T., \& Harabasz, J. (1974). A dendrite method for cluster analysis. Communications in Statistics-Theory and Methods, 3(1), 1-27.

Campedelli, G. M., Aziani, A., \& Favarin, S. (2020). Exploring the effects of covid-19 containment policies on crime: An empirical analysis of the short-term aftermath in los angeles.

Campedelli, G. M., Favarin, S., Aziani, A., \& Piquero, A. R. (2020). Disentangling community-level changes in crime trends during the covid-19 pandemic in chicago. Crime Science, 9(1), 1-18.

Crown Prosecution Service. (2021). 6500 coronavirus-related prosecutions in first six months of the pandemic. https://www.cps.gov.uk/cps/news/6500-coronavirus-related-prosecutions-first-six-monthspandemic

Curman, A. S., Andresen, M. A., \& Brantingham, P. J. (2015). Crime and place: A longitudinal examination of street segment patterns in vancouver, bc. Journal of Quantitative Criminology, 31(1), 127-147. 
Dai, M., Y. Xia \& R. Han. 2021. The impact of lockdown on police service calls during the COVID-19 pandemic in China, Policing, doi:10.1093/police/paab007 .

Dixon, A. and G. Farrell. 2021. A Year of COVID-19 and Crime in England and Wales. Statistical Bulletin on Crime and COVID-19, issue 14. University of Leeds.

Dixon, A., Halford, E., \& Farrell, G. (2020). Spatial distributive justice and crime in the COVID-19 pandemic. Statistical Bulletin on Crime and COVID-19, Issue 2, 27 July 2020, University of Leeds.

Dixon, A., \& Tlley, N. (2020). Fly-tipping during the pandemic. Statistical Bulletin on Crime and COVID-19, Issue 13, 26 November 2020, University of Leeds.

Eck, J. E., Gersh, J. S., \& Taylor, C. (2000). Finding crime hot spots through repeat address mapping. Analyzing Crime Patterns: Frontiers of Practice, 49-64.

Estévez-Soto, P. R. (2020). Crime and covid-19: Effect of changes in routine activities in mexico city.

Felson, M., Jiang, S., \& Xu, Y. (2020). Routine activity effects of the covid-19 pandemic on burglary in detroit, march, 2020. Crime Science, 9(1), 1-7.

Genolini, C., Alacoque, X., Sentenac, M., \& Arnaud, C. (2015a). kml and kml3d: R packages to cluster longitudinal data. Journal of Statistical Software, 65(4), 1-34. http://www.jstatsoft.org/v65/i04/

Genolini, C., Alacoque, X., Sentenac, M., Arnaud, C., \& others. (2015b). Kml and kml3d: R packages to cluster longitudinal data. Journal of Statistical Software, 65(4), 1-34.

Genolini, C., \& Falissard, B. (2011). KmL: A package to cluster longitudinal data. Computer Methods and Programs in Biomedicine, 104(3), e112-e121.

Gerell, M., Kardell, J., \& Kindgren, J. (2020). Minor covid-19 association with crime in sweden. Crime Science, 9(1), 1-9.

Griffiths, E., \& Chavez, J. M. (2004). Communities, street guns and homicide trajectories in chicago, 1980-1995: Merging methods for examining homicide trends across space and time. Criminology, 42(4), 941-978.

Halford, E., Dixon, A., Farrell, G., Malleson, N., \& Tilley, N. (2020). Crime and coronavirus: Social distancing, lockdown, and the mobility elasticity of crime. Crime Science, 9(1), 1-12.

Hodgkinson, T., \& Andresen, M. A. (2020). Show me a man or a woman alone and I'll show you a saint: Changes in the frequency of criminal incidents during the covid-19 pandemic. Journal of Criminal Justice, 69, 101706.

Ignatans, D. \& Pease, K. (2015). Distributive justice and the crime drop, in M.A. Andresen and G. Farrell (Eds) The Criminal Act: The Role and Influence of Routine Activity Theory. London: Palgrave Macmillan. (pp.77-87).

Ignatans, D., \& Pease, K. (2016). On whom does the burden of crime fall now? Changes over time in counts and concentration. International Review of Victimology, 22(1), 55-63.

Langton, S., Dixon, A., \& Farrell, G. (2021). Six months in: Pandemic crime trends in england and wales. Crime Science, 10(1), 1-16.

Langton, S., \& Solymosi, R. (2020). Open street map for crime and place. OSF Preprints, https://osf.io/a96y7.

Malleson, N. and M.A. Andresen. 2015. Spatial-temporal crime hotspots and the ambient population, Crime Science, 4(10); 1-8. 
Malleson, N. and M.A. Andresen. 2016. Exploring the impact of ambient population measures on London crime hotspots, Journal of Criminal Justice, 46; 52-63.

McVie, S., Norris, P., \& Pillinger, R. (2020). Increasing inequality in experience of victimization during the crime drop: Analysing patterns of victimization in scotland from 1993 to 2014-15. The British Journal of Criminology, 60(3), 782-802.

Mohler, G., Bertozzi, A. L., Carter, J., Short, M. B., Sledge, D., Tita, G. E., Uchida, C. D., \& Brantingham, P. J. (2020). Impact of social distancing during covid-19 pandemic on crime in los angeles and indianapolis. Journal of Criminal Justice, 101692.

Newton, A. (2018). Macro-level generators of crime, including parks, stadiums, and transit stations.

Office of National Statistics. (2012). Census geography.

https://www.ons.gov.uk/file?uri=/methodology/geography/ukgeographies/censusgeography/changesto outputareasandsuperoutputareas2001to2011tcm77288393.pdf

Office for National Statistics. 2020. Coronavirus and crime in England and Wales: August 2020.

Statistical Bulletin, 26 August 2020. Office for National Statistics.

Office of National Statistics. (2021). Census geography.

https://www.ons.gov.uk/methodology/geography/ukgeographies/censusgeography\#super-output-areasoa on Economics and the Environment

Padgham, M., Lovelace, R., Salmon, M., \& Rudis, B. (2017). Osmdata. Journal of Open Source Software, 2(14).

Payne, J., A Morgan, and A. Piquero (2020). COVID-19 and social distancing measures in Queensland, Australia, are associated with short-term decreases in recorded violent crime, Journal of Experimental Criminology, https://dx.doi.org/10.1007\%2Fs11292-020-09441-y

Payne, J., A. Morgan, and A. Piquero (2021). Exploring regional variability in the short-term impact of COVID-19 on property crime in Queensland, Australia, Crime Science, 10(7); 1-20.

Piquero, A. R., Riddell, J. R., Bishopp, S. A., Narvey, C., Reid, J. A., \& Piquero, N. L. (2020). Staying home, staying safe? A short-term analysis of covid-19 on dallas domestic violence. American Journal of Criminal Justice, 1-35.

R Core Team. (2013). R: A language and environment for statistical computing. Vienna, Austria.

Shayegh, S., \& Malpede, M. (2020). Staying home saves lives, really! Staying Home Saves Lives, Really!

Stickle, B., \& Felson, M. (2020). Crime rates in a pandemic: The largest criminological experiment in history. American Journal of Criminal Justice, 45(4), 525-536.

Tompson, L., Johnson, S., Ashby, M., Perkins, C., \& Edwards, P. (2015). UK open source crime data: Accuracy and possibilities for research. Cartography and Geographic Information Science, 42(2), 97111.

Trickett, A., D. Ellingworth, T. Hope and K. Pease. 1995. Crime victimization in the eighties: Changes in area and regional inequality, British Journal of Criminology, 35(3); 343-359.

Van Dijk, J.J.M., A. Tseloni and G. Farrell (Eds). 2012. The International Crime Drop: New Directions in Research. New York: Palgrave Macmillan. 
